\title{
Elliptical Monopole Antenna on InP Substrate for Sub-THz RTD-based Oscillators
}

\author{
H. M. Santos*†, L. M. Pessoa*, H. M. Salgado*† \\ *INESC TEC \\ ${ }^{\dagger}$ Faculty of Engineering, University of Porto \\ \{hugo.m.santos, lpessoa, hsalgado\}@inesctec.pt
}

\author{
Pedro Pinho \\ Instituto de Telecomunicações \\ ADEETC, Instituto Superior de Engenharia de Lisboa \\ ppinho@deetc.isel.pt
}

\begin{abstract}
The high permittivity of InP substrates has been a limiting factor for the bandwidth and efficiency of antennas fabricated in this material. In this manuscript we propose an elliptical monopole, monolithically fabricated in InP, fed by a CPW line. The suggested topology was simulated using HFSS finite element method. Input reflection coefficient measurements were performed on the monopole to validate the proposed antenna. Simulated and measured $-10 \mathrm{~dB}$ bandwidths of 27 and $24 \mathrm{GHz}$ were obtained, respectively. The peak simulated efficiency and realized gain were $95.37 \%$ and $4.6 \mathrm{dBi}$.
\end{abstract}

\section{INTRODUCTION}

Antennas fabricated on high permittivity materials typically suffer from reduced bandwidth and degraded efficiencies. At sub- $\mathrm{THz}$ frequencies the permittivity of $\mathrm{InP}$ was measured to be $\epsilon_{r}=12.33$ and the loss tangent $\tan \delta=0.009$ [1]. Brown [2] stated that the ratio of radiation which goes in to the substrate and the one that escapes to free space is approximately $\epsilon_{r}^{3 / 2}$. Additionally, he concluded that, if the frequency is within the sub-THz range, substrate modes appear which distort the radiation pattern of antennas and lead to a guided wave being reflected in the top and bottom interfaces.

In 1993, Brown proposed a photonic crystal for GaAs to circumvent substrate modes and enhance the air to substrate radiation ratio. Nonetheless, Brown's work requires a well established but expensive fabrication process. In 2015 Choi proposed a wire array design using three layers of benzocyclobutene (BCB) on top of an InP substrate, which yielded a simulated gain of $5 \mathrm{dBi}$ with a peak radiation efficiency of $70 \%$ [4]. Lee proposed a rectangular cavity antenna from which he attained a maximum radiation efficiency of $70 \%$ and a bandwidth of $41 \mathrm{GHz}$ centred at $300 \mathrm{GHz}$ [5]. In 2016 Choi designed a rectangular cavity antenna for operation at $280 \mathrm{GHz}$, albeit with a relatively low radiation efficiency of $52 \%$ and an impedance bandwidth of $20 \mathrm{GHz}$ [6].

In 1992, Honda reasoned from the symmetry of the disk monopole that it should exhibit broadband omnidirectionality [7]. From this work onwards, planar topologies were proposed for low frequencies and omnidirectional ultra wideband (UWB) applications, where ground plane shaping was used for impedance matching [8]. For sub- $\mathrm{THz}$ frequencies Li suggested the usage of the circular monopole in a photonic transmitter where the high permittivity GaAs substrate beneath the antenna was removed [9].
In this study we propose the usage of an elliptical monopole, monolithically fabricated in InP for easy integration with the resonant tunneling diode (RTD) oscillator. This antenna exhibits a wideband reflection response and is meant to be used as a radiating element for RTD-based oscillators to achieve air-side radiation. Shaping the ground plane of the feeding coplanar waveguide line enables one to attain better impedance matching, radiation pattern stability and satisfactory front-toback ratio for the envisaged application. The paper is organized as follows: the next section addresses the proposed topology and preliminary design; section III focuses on the simulation of the antenna performance on the InP substrate and presentation of measurement results; in section IV the final conclusions and future work guidelines are given.

\section{Antenna Design}

The model of the antenna is represented in Fig. 1. An InP substrate with a height of $100 \mu \mathrm{m}$ and a $400 \mathrm{~nm}$ thick gold metallization were used for designing the monopole. We considered a permittivity of $\epsilon_{r}=12.6$ for the design as given by the fabrication technology. An exponential shaping of the form $y=k \cdot e^{a x}$ was introduced in the ground-plane to improve the bandwidth when compared to a linear cut. The factors $k$ and $a$ are obtained from the $(x, y)$ coordinates of the points that the exponential curve should intersect. The design variables were swept to achieve optimum performance and their final values were obtained as follows: $D=190 \mu \mathrm{m}$, $d=120 \mu \mathrm{m}, g=6 \mu \mathrm{m}, h=137.5 \mu \mathrm{m}, w=51 \mu \mathrm{m}$. An infinite reflector plane was considered below the substrate to obtain air-side radiation.

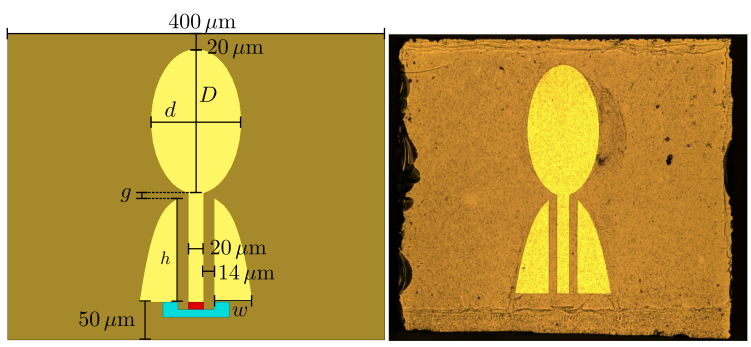

Fig. 1: Antenna model used for finite element method simulation in HFSS (left) and fabricated sample (right). 


\section{RESULTS AND DISCUSSION}

The measurement setup depicted in Fig. 2 was used to characterize the reflection coefficient of the proposed antenna. The antenna sample was placed above a large copper reflector to mimic the simulation model where an infinite reflector plane was used.

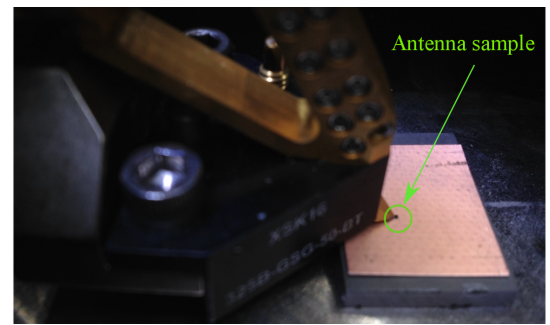

Fig. 2: Measurement setup with probing station, reflector plane and antenna sample.

In Figs. 3 and 4 the measured reflection coefficient magnitude and simulated radiation patterns are shown, respectively. A good agreement can be seen between the measured and the simulated $\left|S_{11}\right|$, despite the presence of some undesired reflections from the measurement setup and a slight offset in the resonant frequency, the latter most likely due to moisture absorption causing InP permittivity to fluctuate. The radiation patterns were verified to remain unaltered throughout the operating bandwidth of the antenna. The simulated peak realized gain and peak efficiency were $4.6 \mathrm{dBi}$ and $95.37 \%$, at $300 \mathrm{GHz}$, respectively.

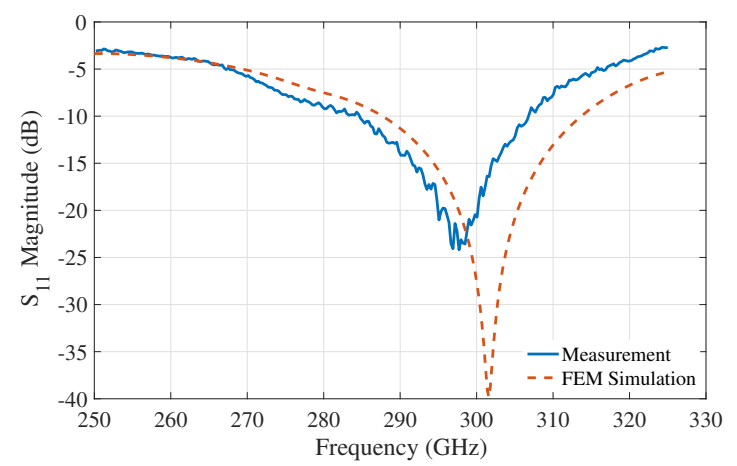

Fig. 3: Measured and simulated $\left|S_{11}\right|$ of elliptical monopole.

\section{CONCLUSION}

In this work a successful implementation of an elliptical monopole in InP for sub- $\mathrm{THz}$ communications was shown. The good agreement between the measurements and the simulation results validated the proposed antenna, which was carefully designed to mitigate substrate modes and achieve air-side radiation. Nonetheless, some variations on the InP permittivity shall be considered on future designs.

The possibility of including this antenna as feeding element of a larger horn antenna is also interesting as the gain can be highly improved, thus increasing the distance at which high throughput communications can occur.

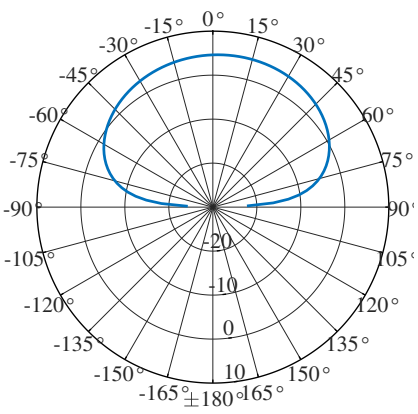

(a)

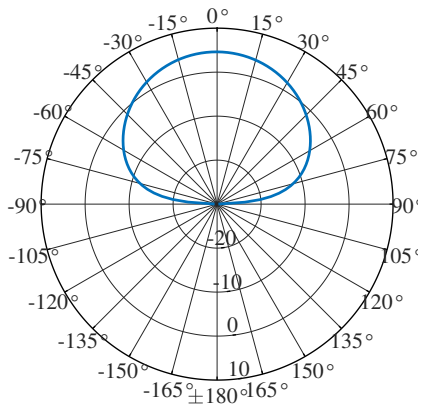

(b)
Fig. 4: Simulated E-plane (a) and H-plane (b) radiation patterns at $300 \mathrm{GHz}$.

\section{ACKNOWLEDGMENT}

The authors would like to thank Edward Wasige, Jue Wang, Abdullah Al-Khalidi and Cui Zhang at the University of Glasgow for providing the antenna fabrication and measurements. This work was supported by the iBROW project (grant agreement no. 645369), supported by the European Commission under the Horizon 2020 Programme, and by FCT (Fundação para a Ciência e a Tecnologia, Portugal), through the Ph.D. grant $\mathrm{PD} / \mathrm{BD} / 113821 / 2015$.

\section{REFERENCES}

[1] J. A. Hejase, P. R. Paladhi, and P. P. Chahal, "Terahertz Characterization of Dielectric Substrates for Component Design and Nondestructive Evaluation of Packages," IEEE Transactions on Components, Packaging and Manufacturing Technology, vol. 1, no. 11, pp. 1685-1694, Nov 2011.

[2] E. R. Brown, C. D. Parker, and E. Yablonovitch, "Radiation properties of a planar antenna on a photonic-crystal substrate," J. Opt. Soc. Am. B, vol. 10, no. 2, pp. 404-407, Feb 1993.

[3] S. Diebold, S. Nakai, K. Nishio, K. Tsuruda, T. Mukai, M. Fujita, and T. Nagatsuma, "Packaging of THz circuits using a HDPE lens and an impedance-matched carrier substrate," in 2015 Asia-Pacific Microwave Conference $(A P M C)$, vol. 1, Dec 2015, pp. 1-3.

[4] S. H. Choi, M. Kim, K. J. Lee, and J. S. You, "A terahertz wire-array antenna integrated on a 75 um InP substrate," in 2015 IEEE International Symposium on Antennas and Propagation USNC/URSI National Radio Science Meeting, July 2015, pp. 2103-2104.

[5] K. M. Lee, I. J. Lee, S. Jeon, M. Kim, and J. S. You, "300 GHz InP rectangular cavity antenna," in 2015 IEEE International Symposium on Antennas and Propagation USNC/URSI National Radio Science Meeting, July 2015, pp. 2105-2106.

[6] M. Kim, S. H. Choi, S. Jeon, and I. J. Lee, “A 280-GHz Rectangular Cavity Antenna Integrated in 80um InP Substrate," IEEE Antennas and Wireless Propagation Letters, vol. PP, no. 99, pp. 1-1, 2016.

[7] S. Honda, M. Ito, H. Seki, and Y. Jinbo, "A disk monopole antenna with 1:8 impedance bandwidth and omnidirectional radiation pattern," in Proceedings of the International Symposium on Antennas and Propagation Japan, vol. 4. Citeseer, 1992, pp. 1145-1145.

[8] Q. Wu, R. Jin, J. Geng, and M. Ding, "Compact CPW-fed quasi-circular monopole with very wide bandwidth," Electronics Letters, vol. 43, no. 2, pp. 69-70, January 2007.

[9] Y. T. Li, J. W. Shi, C. Y. Huang, N. W. Chen, S. H. Chen, J. I. Chyi, Y. C. Wang, C. S. Yang, and C. L. Pan, "Characterization and Comparison of GaAs/AlGaAs Uni-Traveling Carrier and Separated-TransportRecombination Photodiode Based High-Power Sub-THz Photonic Transmitters," IEEE Journal of Quantum Electronics, vol. 46, no. 1, pp. 19-27, Jan 2010 . 